\title{
Unita di consumo dei farmaci e valutazioni farmacoeconomiche: uso e misuso di DDD e PDD
}

Mario Eandi*

\begin{abstract}
In pharmacoeconomical evaluations the quantification of drug utilization has to be done on the basis of measurement units that allow comparisons among series of longitudinal and transversal data. The most common techniques used for the analysis and the comparison of drug utilization patterns are based either on the Defined Daily Dose (DDD), a unit system proposed by WHO's Drug Utilization Research Group, or on the Prescribed Daily Dose (PDD), a statistical parameter obtained from the analysis of drug prescriptions. This article illustrates the meaning of the main indicators of drug consumption that can be build with these techniques, underlining their utility and limitations.

The DDD is the conventionally established theoretical mean daily dose of a drug, referred to a way of administration and to its main indication. It is, therefore, a mere technical measurement unit that cannot be interpreted as mean prescribed or consumed dose, and even less as recommended dose.

The PDD, on the contrary, is not a measurement unit but a statistical mean value, that expresses the central tendency of the prescription variability in a defined setting.

Starting from Italian data on the consumption of wide-spread antibiotics, the use and interpretation of various indicators based on the DDDs and PDDs are discussed. The parameters derived with the DDD technique are suitable for monitoring drug utilization and pharmaceutical expenditure. The PDD method is more direct, indicates the mean quantities actually prescribed and permits the estimation of the total dose consumed per therapeutic cycle and of other clinically relevant parameters, but requires the acquisition of more data than the other technique does.

It is also important to remark the fact that both methods can't be directly used for economical evaluations trying to assess the efficiency of resource allocation, as they are not correlated to the clinical outcomes of the therapy.
\end{abstract}

Farmeconomia e percorsi terapeutici 2002; 3 (4): 209-222

\section{INTRODUZIONE}

Le valutazioni farmacoeconomiche possono avere finalità differenti e possono utilizzare tecniche diverse in funzione degli obiettivi specifici del decisore.

Talora la finalità è quella di analizzare l'andamento della spesa farmaceutica a diversi livelli di aggregazione del sistema sanitario (nazionale, regionale, aziendale, ospedaliera) o per gruppi terapeutici o per singoli farmaci. In questo caso una semplice analisi dei costi d'acquisto dei farmaci prescritti, dispensati o realmente consumati può essere adeguata per soddisfare le istanze del decisore. La rilevazione dei farmaci prescritti, dispensati o consumati può essere attuata con varie tecniche epidemiologiche, estraendo informazioni a vari livelli della catena distributiva; solo in casi particolari si ottengono dati di utilizzo estraendoli direttamente dalla storia clinica del singolo paziente: generalmente questo avviene nel corso di studi clinici tarati su obiettivi specifici e limitati a singoli farmaci o a gruppi di farmaci omogenei.

In ogni caso, la quantificazione dei consumi o dell'utilizzazione dei farmaci deve essere attuata mediante unità di misura che permettano un confronto tra serie di dati trasversali (ad esempio, confronto tra nazioni, regioni, ASL, divisioni ospedaliere) e longitudinali (ad esempio, confronto tra anni successivi). Le tecniche più frequentemente utilizzate per analizzare e confrontare il consumo dei farmaci sono basate su alcuni indicatori costruiti attorno al sistema di unità di misure, denominato Defined Daily Doses (DDD), o attorno al parametro statistico, conosciuto come Prescribed Daily Doses (PDD).

\author{
* Farmacologia \\ Clinica, Università di \\ Torino
}


In questo lavoro intendiamo discutere il significato dei principali indicatori di farmacoutilizzazione ricavabili con le tecniche delle DDD e delle PDD, sottolineandone utilità e limiti mediante esemplificazioni costruite sul confronto dei dati di utilizzo di alcuni antibiotici di ampia diffusione in ambito territoriale ed ospedaliero.

\section{DEFINED DAILY DOSES (DDD) e PRESCRIBED DAILY DOSES (PDD)}

Fin dai primi studi pionieristici sull'utilizzazione dei farmaci è emerso che esisteva una considerevole variabilità nelle modalità di consumo sia tra nazioni sia all'interno di una stessa nazione. [1-3] Inoltre, differenze nella classificazione dei farmaci e nelle unità di misura rendevano problematico il confronto tra le modalità di utilizzo dei farmaci nei vari paesi o all'interno degli stessi. Per risolvere questi problemi e standardizzare i metodi di valutazione, il gruppo di studio dell'OMS, WHO Drug Utilization Research Group (DURG), propose di usare la tecnica delle Defined Daily Dose (DDD) per convertire i diversi pattern di consumo dei farmaci in unità confrontabili. [2-9]

La DDD è l'ipotetica dose media giornaliera di mantenimento di un farmaco, riferita ad una via di somministrazione e all'indicazione terapeutica principale per l'adulto. $[3,4,6,7]$

La DDD viene indicata generalmente in peso di principio attivo (grammi), quando nel prodotto farmaceutico è incluso un solo principio attivo, oppure in unità di somministrazione (ED da Enkelt Dose, o dose singola in norvegese) qualora il prodotto contenga più principi attivi o principi attivi quantificabili in unità biologiche.

La DDD è un'unità di misura stabilita in modo convenzionale per quantificare e confrontare i consumi di un farmaco o di gruppi di farmaci omogenei per livello di classificazione secondo il sistema ATC. [5-8, 10-15]

Occorre sottolineare con grande evidenza come la DDD sia un'unità tecnica di misura e di confronto, alla quale non si può, né si deve, attribuire il significato di dose media prescritta od utilizzata e tanto meno di dose raccomandata.

Mediante l'unità di misura DDD si possono stimare le quantità di farmaco utilizzate, esprimendole in numero di DDD piuttosto che in numero di grammi: gli indicatori basati sul numero di DDD consumate servono a quantificare l'intensità di utilizzo di un farmaco o di gruppo di farmaci in un dato contesto di analisi. [6]

Occorre, inoltre, precisare come i valori delle DDD attribuiti a farmaci diversi, ma ap- partenenti ad uno stesso gruppo terapeutico, non possano e non debbano essere interpretati come dosi equiefficaci o terapeuticamente equivalenti. Pertanto, rappresenta un grave errore metodologico utilizzare il costo di una DDD in analisi farmacoeconomiche comparative basate sulla tecnica "minimizzazione dei costi”.

L'unità di misura DDD è definibile teoricamente per ogni farmaco e per ogni principale via di somministrazione. In realtà la lista delle DDD non è mai completa e viene definita in linea prioritaria per i farmaci più importanti e per la via orale e parenterale.

La prima lista dei valori delle DDD è stata pubblicata in Norvegia dal Nordic Council on Medicines. Successivamente il metodo delle DDD è stato fatto proprio dall' Organizzazione Mondiale della Sanità (OMS) ed integrato con il sistema di classificazione ATC. [7-9]

Oggigiorno, le DDD vengono assegnate per i farmaci classificati secondo il $5^{\circ}$ livello ATC dal gruppo di esperti internazionali incaricati dal WHO Collaborating Centre for Drug Statistics Methodology in Norvegia. [8,9] Per certi tipi di farmaco non sono state assegnate le DDD o perché è difficile trovare una dose appropriata o perché nessuno ne ha fatto richiesta al Centro dell' OMS.

In diversi paesi europei, tra cui l'Italia, esistono gruppi di lavoro (comitati locali) che hanno il compito di adattare il sistema delle DDD in relazione ai farmaci disponibili nelle rispettive nazioni. [15]

L'uso delle DDD negli studi di farmacoepidemiologia si è diffuso prevalentemente nei paesi europei. Negli Stati Uniti si preferisce utilizzare le Prescribed Daily Doses, ovvero esprimere la quantità di farmaco utilizzata come dose media giornaliera prescritta, indicata direttamente in unità ponderali (grammi) piuttosto che in unità convenzionali DDD. [12]

A differenza della DDD, la PDD non è un'unità di misura, ma un valore medio statistico che esprime la tendenza centrale della variabilità del comportamento prescrittivo rilevato in un ambito predefinito di indagine.

\section{GLI INDICATORI STIMABILI CON LA TECNICA DELLE DDD}

Il sistema delle DDD è un sistema di unità di misure studiato e messo a punto per facilitare l'analisi comparativa delle modalità prescrittive e/o dell'intensità di consumo dei farmaci, svincolando la stima quantitativa dei consumi dai parametri fisici del prodotto commerciale (tipo di formulazione farmaceutica, quantità per unità farmaceutica, numero di unità per confezioni). La tecnica delle DDD con- 
sente di sommare omogeneamente i consumi correlati a singoli principi attivi o a singole classi terapeutiche di farmaci, raggruppati secondo i livelli ATC. [7-9]

L'unità di misura DDD viene usata per costruire diversi indicatori dell'intensità d'utilizzo dei farmaci, partendo dalla rilevazione dei consumi o delle prescrizioni.

I principali indicatori basati sulle DDD sono descritti e definiti nel riquadro A.

La tecnica delle DDD viene applicata per analizzare dati di vendita o di prescrizione dei farmaci, ottenuti mediante indagini campionarie. La qualità dell'informazione campionata varia sensibilmente nei due casi.

La quantità di vendite viene rilevata costantemente da un'agenzia multinazionale privata (IMS) attraverso una rete di rilevatori distribuiti sul territorio delle diverse nazioni: la rilevazione delle vendite avviene a livello di farmacie e di grossisti. Utilizzando validati modelli statistici, dalle rilevazioni campionarie opportunamente dimensionate si estrapolano le vendite totali del singolo prodotto commerciale nel periodo di tempo considerato, esprimendole sia come volume (numero di confezioni) sia come valore monetario.
Quando le informazioni disponibili consistono unicamente nel numero di confezioni vendute in un dato intervallo di tempo, è possibile stimare solo due indicatori basati sulla tecnica delle DDD: il Numero di DDD/1000 abitanti/anno ed il Numero di DDD/1000 abitanti/ giorno.

Un esempio della costruzione di questi due indicatori è riportato nella tabella 1 per alcuni antibiotici di largo uso.

Immaginiamo di avere rilevato e stimato il numero di confezioni per uso orale vendute per ciascun prodotto (i dati utilizzati sono in realtà ricavati indirettamente da valori di prescrizione e non di vendita).

Alcuni antibiotici sono disponibili esclusivamente a un livello dose, altri a due o tre livelli dose. Le confezioni di ciascun prodotto variano come numero di unità farmaceutiche presenti e quindi come quantità complessiva in peso. Conoscendo il numero di confezioni vendute si stima il numero di unità farmaceutiche (dosi) consumate. Considerando il valore della DDD per via orale assegnato a ciascun antibiotico, possiamo stimare per ogni prodotto farmaceutico il numero di unità dose necessarie per realizzare una DDD e quindi il numero cumulativo di DDD consumate in un anno sul-

\begin{tabular}{|c|c|c|c|c|c|c|c|c|}
\hline & $\begin{array}{c}\text { N. } \\
\text { dosi/conf }\end{array}$ & $\begin{array}{c}\text { N. } \\
\text { Confezioni } \\
\text { vendute/anno }\end{array}$ & $\begin{array}{l}\text { N. Unità } \\
\text { dose/anno }\end{array}$ & $\begin{array}{l}\text { Valore } \\
\text { DDD } \\
\text { (mg) }\end{array}$ & $\begin{array}{c}\text { N. } \\
\text { Unita/DDD }\end{array}$ & $\begin{array}{c}\text { N. DDD } \\
\text { cumulative/anno }\end{array}$ & $\begin{array}{c}\text { N. } \\
\text { DDD/1000ab/anno }\end{array}$ & $\begin{array}{c}\text { N. } \\
\text { DDD/1000ab/die }\end{array}$ \\
\hline \multicolumn{9}{|l|}{ CIPROFLOXACINA } \\
\hline $\begin{array}{l}\text { CPR RIV } 250 \mathrm{mg} \\
\text { CPR RIV 500mg } \\
\text { CPR RIV 750mg } \\
\text { Totale }\end{array}$ & $\begin{array}{c}10 \\
6 \\
12\end{array}$ & $\begin{array}{c}1.320 .150 \\
2.795 .880 \\
115.910 \\
4.231 .940\end{array}$ & $\begin{array}{c}13.201 .500 \\
16.775 .280 \\
1.390 .920 \\
31.367 .700\end{array}$ & $\begin{array}{l}1000 \\
1000 \\
1000\end{array}$ & $\begin{array}{c}4 \\
2 \\
1,3\end{array}$ & $\begin{array}{c}3.300 .375 \\
8.387 .640 \\
1.043 .190 \\
12.731 .205\end{array}$ & $\begin{array}{c}57,06 \\
145,00 \\
18,03 \\
220,10\end{array}$ & $\begin{array}{l}0,16 \\
0,40 \\
0,05 \\
0,60\end{array}$ \\
\hline \multicolumn{9}{|l|}{ LEVOFLOXACINA } \\
\hline $\begin{array}{l}\text { CPR RIV } 250 \mathrm{mg} \\
\text { CPR RIV 500mg } \\
\text { Totale }\end{array}$ & $\begin{array}{l}5 \\
5\end{array}$ & $\begin{array}{c}923.976 \\
2.866 .132 \\
3.790 .108\end{array}$ & $\begin{array}{l}4.619 .880 \\
14.330 .660 \\
18.950 .540\end{array}$ & $\begin{array}{l}250 \\
250\end{array}$ & $\begin{array}{c}1 \\
0,5\end{array}$ & $\begin{array}{l}4.619 .880 \\
28.661 .320 \\
33.281 .200\end{array}$ & $\begin{array}{c}79,87 \\
495,49 \\
575,36\end{array}$ & $\begin{array}{l}0,22 \\
1,36 \\
1,58\end{array}$ \\
\hline \multicolumn{9}{|l|}{ MOXIFLOXACINA } \\
\hline CPR 400mg & 5 & 741.936 & 3.709 .680 & 400 & 1 & 3.709 .680 & 64,13 & 0,18 \\
\hline \multicolumn{9}{|l|}{ NORFLOXACINA } \\
\hline CPR 400mg & 14 & 1.002 .589 & 14.036 .250 & 800 & 2 & 7.018 .125 & 121,33 & 0,33 \\
\hline \multicolumn{9}{|l|}{ CLARITROMICINA } \\
\hline $\begin{array}{l}\text { CPR RIV 250mg } \\
\text { CPR RIV 500mg } \\
\text { CPR RM 500mg } \\
\text { Totale }\end{array}$ & $\begin{array}{c}12 \\
14 \\
7\end{array}$ & $\begin{array}{c}802.560 \\
858.311 \\
308.309 \\
1.969 .180\end{array}$ & $\begin{array}{c}9.630 .720 \\
12.016 .360 \\
2.158 .160 \\
23.805 .240\end{array}$ & $\begin{array}{l}500 \\
500 \\
500\end{array}$ & $\begin{array}{l}2 \\
1 \\
1\end{array}$ & $\begin{array}{c}4.815 .360 \\
12.016 .360 \\
2.158 .160 \\
18.989 .880\end{array}$ & $\begin{array}{c}83,25 \\
207,74 \\
37,31 \\
328,29\end{array}$ & $\begin{array}{l}0,23 \\
0,57 \\
0,10 \\
0,90\end{array}$ \\
\hline \multicolumn{9}{|l|}{ KETOLIDE } \\
\hline CPR RIV 400mg & 10 & 159.900 & 1.599 .000 & 800 & 2 & 799.500 & 13,82 & 0,04 \\
\hline
\end{tabular}

\section{Tabella 1}

Esempio di costruzione degli indicatori di intensità di utilizzazione di alcuni antibiotici secondo il sistema DDD utilizzando dati di vendita stimati in Italia nell'anno 03/2001-03/2002 e assumendo una popolazione complessiva di 57.844.000 abitanti. 
l'intero territorio nazionale per ogni prodotto e complessivamente per ogni antibiotico.

Infine, conoscendo il numero di abitanti nel territorio considerato, si possono calcolare i due indicatori di intensità d'utilizzo sopra descritti.

I dati riportati nella tabella 1 evidenziano che il N. DDD/1000 abitanti/anno stimato per ogni prodotto e, cumulativamente, per ogni antibiotico considerato, è sempre relativamente basso e porterebbe a concludere che un ciclo di terapia antibiotica dura mediamente meno di un giorno. Ovviamente tale conclusione è assurda e il risultato numerico ottenu-

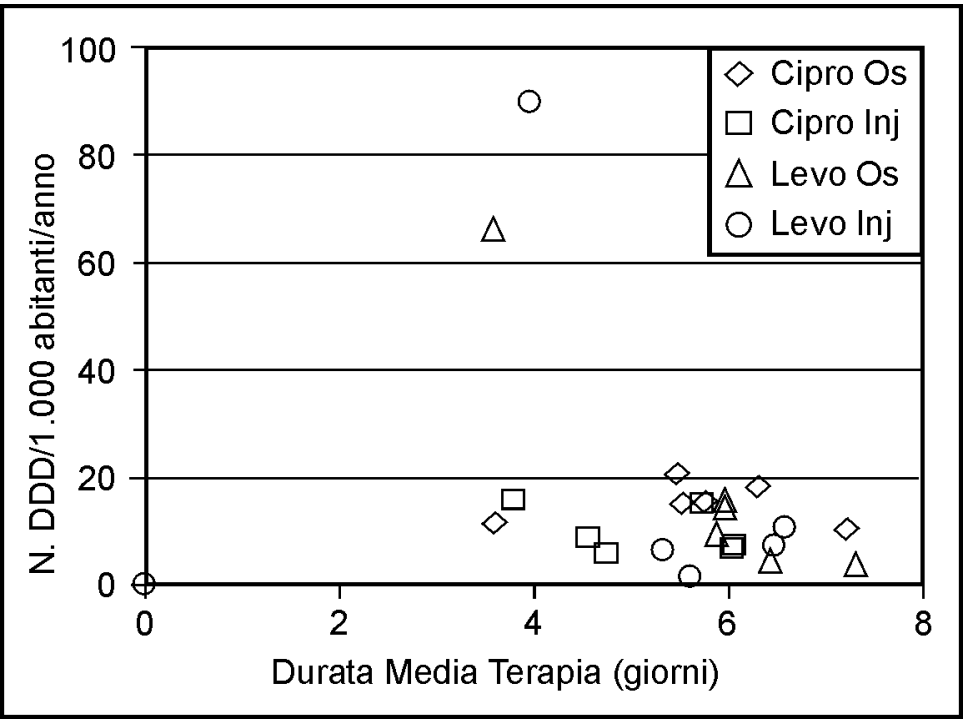

Figura 1

Rappresentazione grafica della relazione tra durata media della terapia ed il numero di DDD/1000 abitanti/anno. Dati relativi ai consumi di sei nazioni nell'anno 2001

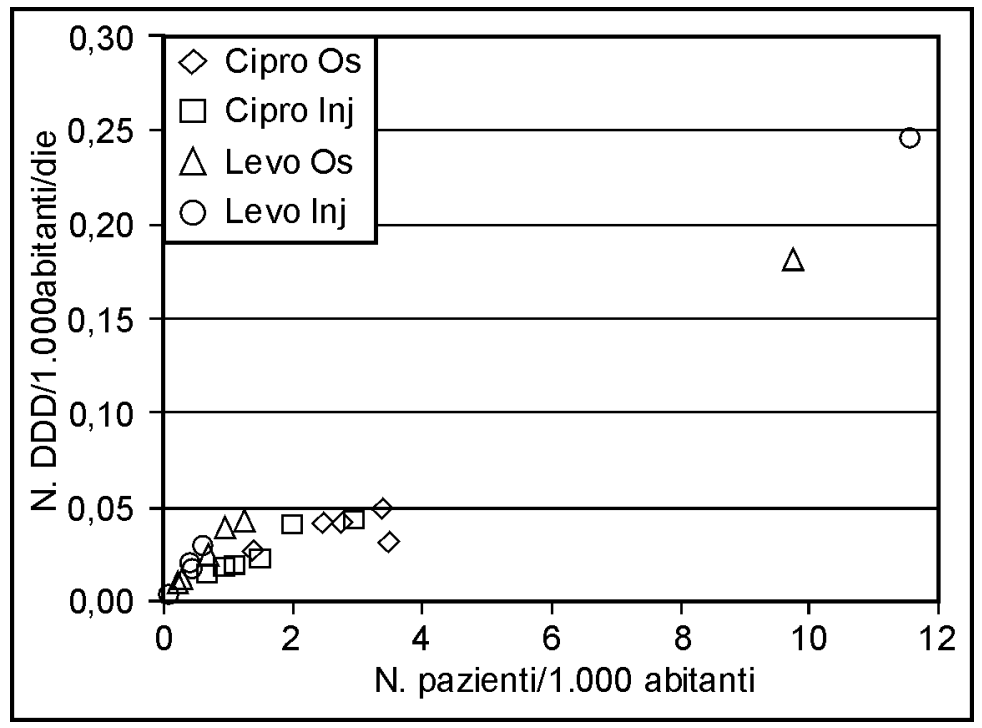

Figura 2

Rappresentazione grafica della relazione tra N. pazienti/anno/1000 abitanti e numero di DDD/1000 abitanti/giorno. Dati relativi ai consumi di sei nazioni nell'anno 2001. to dipende dal fatto che solo una piccola percentuale della popolazione viene trattata col farmaco. D'altra parte il N. DDD/1000 abitanti/ giorno indica che una percentuale relativamente bassa della popolazione viene trattata ogni anno con i singoli prodotti e, cumulativamente, con i singoli antibiotici.

Per valutare il grado di affidabilità e robustezza del numero di DDD/1000 abitanti/anno nello stimare l'intensità di utilizzo di un antibiotico abbiamo cercato di verificare se esista una correlazione rettilinea tra questo indicatore e la durata media di un ciclo di trattamento. Per attuare tale verifica abbiamo utilizzato un particolare insieme di dati relativi all'utilizzo della ciprofloxacina e della levofloxacina, orali e parenterali, rilevati nel 2001 in sei paesi industrializzati: Italia, Francia, Spagna, Germania, Regno Unito e Stati Uniti. Questo insieme di dati, stratificati per via di somministrazione e per nazione, comprendeva la quantità di vendite annuali e la durata media dei trattamenti. La figura 1 rappresenta graficamente la correlazione tra il numero di DDD/1000 abitanti/anno della ciprofloxacina e della levofloxacina orali e parenterali e le relative durate medie (giorni) di trattamento nei 6 paesi analizzati. Non abbiamo potuto evidenziare alcuna correlazione rettilinea tra queste due variabili. (Figura 1)

Dobbiamo concludere che il numero di DDD/1000 abitanti/anno è un indicatore che, nel caso esaminato, non rappresenta in modo affidabile la durata media del trattamento farmacologico.

Questo risultato indica che il Numero di DDD/1000 abitanti/anno è un indicatore non particolarmente utile e non sufficientemente robusto per confrontare l'intensità di utilizzazione di antibiotici diversi, mentre è sufficientemente adeguato per analizzare le variazioni di utilizzazione di uno stesso antibiotico in aree geografiche $o$ assistenziali differenti o in periodi successivi.

Per ovviare almeno in parte a questi inconvenienti, recentemente sono state proposte alcune integrazioni alla tecnica delle DDD. $[16,17]$

Con il medesimo insieme di dati abbiamo anche valutato il grado di affidabilità e di robustezza del numero di DDD/1000 abitanti/ giorno nello stimare la proporzione di soggetti di una popolazione trattati annualmente col farmaco. Per attuare questa verifica abbiamo correlato il numero di DDD/1000 abitanti/giorno di ciascun antibiotico con la proporzione di soggetti trattati con il farmaco in ognuna delle nazioni considerate. La figura 2 evidenzia che i due parametri correlano in modo sostanzialmente rettilineo. 
Il numero di DDD/1000 abitanti/giorno, nel caso degli antibiotici considerati, appare dunque come un indicatore abbastanza affidabile dell'intensità d'utilizzo espressa come proporzione di abitanti che annualmente viene trattata col farmaco. Tuttavia, anche questo indicatore deve essere usato con cautela per confrontare l'utilizzo di farmaci diversi o di vie di somministrazioni differenti. Infatti, la figura 2 evidenzia che la regressione rettilinea tra numero di DDD/1000 abitanti/giorno e percentuale di soggetti trattati in una popolazione presenta dispersioni e pendenze differenti nei due antibiotici e nelle due vie di somministrazione considerate.

In conclusione, il numero di DDD/1000 abitanti/anno ed il numero di DDD/1000 abitanti/ giorno sono indicatori che possono essere utili per analizzare le modificazioni dell'intensità d'utilizzo di uno stesso prodotto o di uno stesso antibiotico in aree territoriali o assistenziali diverse o in periodi di tempo (anni) successivi, ma sono poco adatti per confrontare modalità d'utilizzo di antibiotici diversi.

\section{LAPDD COME PARAMETRO DI CONSUMO MEDIO}

La prescrizione dei farmaci viene rilevata su un campione di ricette, opportunamente dimensionato e distribuito sul territorio in modo da essere rappresentativo del comportamento medio dell'intera categoria dei medici e dell'intero mercato dei farmaci. La lettura delle ricette permette di rilevare il numero di confezioni prescritte e dispensate in un intervallo di tempo e quindi di stimare la relativa quantità cumulativa in grammi o in unità DDD. Mediante questo sistema di indagine è possibile rilevare anche le posologie prescritte dai medici e quindi stimare il numero cumulativo di giorni di terapia consumati in un dato intervallo di tempo (un anno). Rapportando la quantità cumulativa di farmaco (grammi) prescritta in un anno al numero di giorni cumulativi di terapia consumati in un anno, si ricava facilmente il valore della PDD del farmaco, relativamente all'ambito di utilizzo considerato. Analogamente, se la quantità cumulativa è espressa in DDD, si ottiene il Numero di DDD/ giorno di trattamento (vedi riquadro A).

La rilevazione delle prescrizioni su un campione di ricette consente anche di stimare il numero di pazienti trattati con il singolo prodotto. Conoscendo i giorni di trattamento e il numero di pazienti trattati si può stimare anche la durata media di un ciclo di terapia con il singolo prodotto o con il singolo farmaco (vedi riquadro $\mathrm{B}$ ).
Le PDD e la durata media (giorni) di terapia sono indicatori molto utili non solo per analizzare il trend del mercato farmaceutico, ma anche per evidenziare e confrontare comportamenti prescrittivi di uno o più farmaci in relazione ad aree territoriali diverse o ad intervalli di tempo successivi.

In alcuni sistemi di rilevazione attraverso le ricette è possibile ottenere informazioni stratificate per indicazioni terapeutiche, ma non è mai possibile ottenere informazioni che riguardano gli esiti delle terapie.

Utilizzando i dati di prescrizione ricavati dalle ricette e conoscendo il numero di abitanti dell'area geografica considerata è anche possibile stimare gli indicatori previsti dalla tecnica delle DDD e in particolare stimare il numero di DDD/1000 abitanti/anno e il numero di DDD/1000 abitanti/giorno. Tuttavia, potendo stimare il valore delle PDD e dei parametri correlati, perde molto interesse l'utilizzo della tecnica delle DDD.

Alcuni programmi di rilevazione sono finalizzati all'analisi delle prescrizioni in ambito ospedaliero: in questo caso è possibile rilevare informazioni relative alle modalità di trattamento (es. vie e modalità, numero di somministrazioni giornaliere, schemi posologici complessi, associazioni di farmaci, ambito di cura, ed eventualmente anche esiti del trattamento). L'analisi del consumo dei farmaci, anche in questo caso, parte dalla rilevazione delle unità farmaceutiche prescritte (più raramente delle confezioni prescritte) e richiede l'adozione di una procedura che permetta di sommare la quantità di farmaco consumata da ogni paziente per ciclo terapeutico o per ricovero. Alcuni autori preferiscono utilizzare la tecnica delle DDD, altri preferiscono esprimere le quantità utilizzate direttamente in unità ponderali e quindi utilizzare la PDD e la durata media di trattamento.

Un esempio di stima delle PDD di alcuni antibiotici ricavate dalle prescrizioni rilevate in Italia nel corso di un anno è riportato nella tabella 2. Si tratta della stessa rilevazione dei consumi già utilizzata per il calcolo degli indicatori basati sulla tecnica delle DDD riportati nella precedente tabella 1 . In questo caso, tuttavia, il calcolo delle PDD parte dalla rilevazione del numero cumulativo di unità farmaceutiche prescritte in un anno per ciascun prodotto e dalla media di somministrazioni giornaliere. Dal rapporto tra questi due valori si ottiene una stima dei giorni cumulativi di trattamento in un anno con i singoli prodotti farmaceutici.

Per ogni prodotto considerato e, cumulativamente, per ogni antibiotico considerato, il 
valore della PDD corrisponde al rapporto tra numero di grammi cumulativi prescritti in un anno e numero di giorni cumulativi di trattamento in un anno.

Si deve innanzitutto notare come le informazioni ottenute dalla rilevazione campionaria delle ricette (numero di confezioni o di dosi prescritte e media del numero di somministrazioni giornaliere) siano più ricche rispetto al caso precedente che disponeva del solo volume di vendite del prodotto. I dati rilevati contengono già preziose informazioni circa le modalità prescrittive degli antibiotici considerati. In particolare è interessante osservare come la media del numero di somministrazioni giornaliere rilevata per alcuni antibiotici si discosti nella pratica clinica dal valore teorico. Ad esempio, nel caso della ciprofloxacina, la media del numero di somministrazioni giornaliere è tendenzialmente inferiore a 2 , valore teorico richiesto per questo antibiotico nella maggior parte delle infezioni, mentre nel caso della levofloxacina la media del numero di somministrazioni giornaliere è tendenzialmente superiore ad 1: evidentemente i medici italiani, in un consistente numero di occasioni, ritengono di dover utilizzare la levofloxacina più di una volta al giorno $\mathrm{e}$, al contrario, di poter somministrare la ciprofloxacina solo una volta al giorno invece di due.

La media del numero di somministrazioni giornaliere rilevate per la moxifloxacina, la norfloxacina, il ketolide e i tre prodotti di claritromicina rispecchiano maggiormente il ritmo posologico teorico indicato per ciascun prodotto.

Confrontando il valore delle PDD stimate per ciascun prodotto e per ciascun antibiotico (tabella 2) con il valore teorico della DDD assegnato ai singoli antibiotici considerati, possiamo notare come nel caso della moxifloxacina, norfloxacina e ketolide via sia una sostanziale concordanza, mentre nel caso della ciprofloxacina, levofloxacina e claritromicina vi sia una significativa discordanza.

Questa discrepanza tra valore teorico della DDD e valore reale delle PDD stimate, particolarmente accentuato quando l'antibiotico è disponibile e usato a più livelli dose, può essere spiegato soprattutto con il fatto che questi antibiotici vengono usati per svariati tipi di infezioni, che richiedono posologie spesso molto diverse da quella ritenuta normale per l'indicazione principale assunta come riferimento per assegnare il valore della DDD.

\begin{tabular}{|c|c|c|c|c|c|c|c|}
\hline & $\begin{array}{l}\text { N. unità dose } \\
\text { prescritte/anno }\end{array}$ & $\begin{array}{l}\text { Media N. } \\
\text { dosi/die }\end{array}$ & $\begin{array}{l}\text { N. Grammi } \\
\text { cumulativi/anno }\end{array}$ & $\begin{array}{c}\text { N. Giorni } \\
\text { Terapia/anno }\end{array}$ & $\begin{array}{c}\text { PDD } \\
\text { Media } \\
\text { (grammi) }\end{array}$ & $\begin{array}{c}\text { N. DDD } \\
\text { cumulative/anno }\end{array}$ & $\begin{array}{c}\text { N. DDD/giorno } \\
\text { terapia }\end{array}$ \\
\hline \multicolumn{8}{|l|}{ CIPROFLOXACINA } \\
\hline CPR RIV 250mg & 13.201 .500 & 1,95 & 3.300 .375 & 6.770 .000 & 0,49 & 3.300 .375 & 0,49 \\
\hline CPR RIV 500mg & 16.775 .280 & 1,84 & 8.387 .640 & 9.117 .000 & 0,92 & 8.387 .640 & 0,92 \\
\hline CPR RIV 750mg & 1.390 .920 & 1,73 & 1.043 .190 & 804.000 & 1,30 & 1.043 .190 & 1,30 \\
\hline Totale & 31.367 .700 & & 12.731 .205 & 16.691 .000 & 0,76 & 12.731 .205 & 0,76 \\
\hline \multicolumn{8}{|l|}{ LEVOFLOXACINA } \\
\hline CPR RIV 250mg & 4.619 .880 & 1,23 & 1.154 .970 & 3.756 .000 & 0,31 & 4.619 .880 & 1,23 \\
\hline CPR RIV 500mg & 14.330 .660 & 1,13 & 7.165 .330 & 12.682 .000 & 0,57 & 28.661 .320 & 2,26 \\
\hline Totale & 18.950 .540 & & 8.320 .300 & 16.438 .000 & 0,51 & 33.281 .200 & 2,02 \\
\hline \multicolumn{8}{|l|}{ MOXIFLOXACINA } \\
\hline CPR 400mg & 3.709 .680 & 1,04 & 1.483 .872 & 3.567 .000 & 0,42 & 3.709 .680 & 1,04 \\
\hline \multicolumn{8}{|l|}{ NORFLOXACINA } \\
\hline CPR 400mg & 14.036 .250 & 1,97 & 5.614 .500 & 7.125 .000 & 0,79 & 7.018 .125 & 0,99 \\
\hline \multicolumn{8}{|l|}{ CLARITROMICINA } \\
\hline CPR RIV 250mg & 9.630 .720 & 1,98 & 2.407 .680 & 4.864 .000 & 0,50 & 4.815 .360 & 0,99 \\
\hline CPR RIV 500mg & 12.016 .360 & 1,94 & 6.008 .180 & 6.194 .000 & 0,97 & 12.016 .360 & 1,94 \\
\hline CPR RM 500mg & 2.158 .160 & 1,06 & 1.079 .080 & 2.036 .000 & 0,53 & 2.158 .160 & 1,06 \\
\hline Totale & 23.805 .240 & & 9.494 .940 & 13.094 .000 & 0,73 & 18.989 .880 & 1,45 \\
\hline \multicolumn{8}{|l|}{ KETOLIDE } \\
\hline CPR RIV 400mg & 1.599 .000 & 2,05 & 639.600 & 780.000 & 0,82 & 799.500 & 1,03 \\
\hline
\end{tabular}

\section{Tabella 2}

Esempio di calcolo delle PDD di alcuni antibiotici stimate in base alle prescrizioni rilevate in Italia nell'anno 03/2001-03/2002 e confronto con analogo indicatore di consumo giornaliero basato sulle DDD. 
Considerando il valore della PDD media di ciascun antibiotico, osserviamo come quello della ciprofloxacina sia inferiore di circa il $25 \%$, quello della claritromicina sia superiore di circa il $50 \%$ e quello della levofloxacina sia supe riore di circa il $100 \%$ rispetti ai valori delle relative DDD.

Questo risultato conferma con i fatti ciò che già dovrebbe essere chiaro per semplice coerenza logica:

il valore della DDD è il valore di un' unità convenzionale di misura utilizzabile per quantificare il consumo di un farmaco, ma in nessun modo può essere confuso con la quantità media di farmaco consumata giornalmente 0 con la dose media raccomandabile.

La quantità di farmaco mediamente prescritta o consumata per giorno di terapia può essere espressa, oltre che in unità ponderali come avviene nelle PDD, anche in unità convenzionali DDD, utilizzando appunto uno degli indicatori sopra descritti e precisamente il N. DDD/giorno trattamento. La tabella 2 nell'ultima colonna a destra riporta i valori di tale indicatore stimati per ciascun prodotto e cumulativamente per ciascun antibiotico.

Confrontando il valore numerico delle PDD con i rispettivi numeri di DDD/giorno di trattamento si può facilmente osservare come il valore di quest'ultimo parametro sia il valore del rapporto tra PDD e DDD: entrambi i parametri, sia pure con valori numerici differenti, hanno un analogo valore informativo sull' intensità del consumo giornaliero di un prodotto o di un farmaco.
La PDD media e ancor più la quantità di farmaco (espressa in unità ponderali o in unità DDD) mediamente consumata in un ciclo di terapia sono i parametri che più fedelmente rappresentano l'intensità di utilizzo dei farmaci in relazione ai bisogni clinici di una popolazione, consentendo di attuare confronti affidabili trasversali e longitudinali.

Un esempio di confronto transnazionale e longitudinale è riassunto nella tabella 3 , che riporta i valori delle quantità medie di ciprofloxacina o di levofloxacina, orali e parenterali, consumate durante un ciclo di terapia in 5 nazioni europee (Italia, Spagna, Francia, Germania e Regno Unito) e negli Stati Uniti in tre anni successivi (1999-2001).

In Italia, Spagna e Germania le quantità medie di ciprofloxacina e di levofloxacina orali per ciclo terapeutico sono sostanzialmente equivalenti, mentre sono sensibilmente inferiori nel Regno Unito e soprattutto negli Stati Uniti. In Spagna si osserva una generale tendenza a utilizzare maggiori quantità di fluorochinoloni parenterali per ciclo di terapia.

La variabilità della quantità di antibiotico consumata mediamente in un ciclo di terapia orale o parenterale riflette le differenze della durata media del ciclo e le differenze delle PDD medie.

L'analisi comparativa delle PDD e delle DDD stimate sullo stesso insieme campionario (dati on-file, qui non riportati) indica che in Italia, Germania, Regno Unito e Stati Uniti vi è una tendenza ad utilizzare la ciprofloxacina orale a posologie giornaliere mediamente inferio-

\begin{tabular}{|c|c|c|c|c|c|c|c|c|c|c|c|c|}
\hline & \multicolumn{4}{|c|}{1999} & \multicolumn{4}{|c|}{2000} & \multicolumn{4}{|c|}{2001} \\
\hline & \multicolumn{2}{|c|}{ Orale } & \multicolumn{2}{|c|}{ Iniettabile } & \multicolumn{2}{|c|}{ Orale } & \multicolumn{2}{|c|}{ Iniettabile } & \multicolumn{2}{|c|}{ Orale } & \multicolumn{2}{|c|}{ Iniettabile } \\
\hline & Gr. & N.DDD & Gr. & N.DDD & Gr. & N.DDD & Gr. & N.DDD & Gr. & N.DDD & Gr. & N.DDD \\
\hline \multicolumn{13}{|l|}{ ITALIA } \\
\hline Ciprofloxacina & 5,29 & 5,29 & 2,70 & 5,39 & 5,32 & 5,32 & 2,87 & 5,74 & 5,43 & 5,43 & 3,46 & 6,93 \\
\hline Levofloxacina & 3,60 & 14,40 & 8,25 & 33,00 & 3,34 & 13,36 & 4,00 & 16,00 & 3,67 & 14,67 & 4,38 & 17,50 \\
\hline \multicolumn{13}{|l|}{ SPAGNA } \\
\hline Ciprofloxacina & 6,53 & 6,53 & 3,96 & 7,92 & 5,62 & 5,62 & 4,17 & 8,33 & 5,94 & 5,94 & 3,55 & 7,09 \\
\hline Levofloxacina & 3,43 & 13,71 & 4,17 & 16,67 & 3,00 & 12,00 & 4,40 & 17,60 & 3,24 & 12,97 & 4,15 & 16,62 \\
\hline \multicolumn{13}{|l|}{ FRANCIA } \\
\hline Ciprofloxacina & 7,49 & 7,49 & 3,01 & 6,02 & 7,34 & 7,34 & 2,98 & 5,96 & 7,06 & 7,06 & 3,09 & 6,17 \\
\hline Levofloxacina & ---- & ---- & ---- & ---- & ---- & ---- & ---- & ---- & 4,00 & 16,00 & 3,20 & 12,80 \\
\hline \multicolumn{13}{|l|}{ GERMANIA } \\
\hline Ciprofloxacina & 5,09 & 5,09 & 2,77 & 5,54 & 5,09 & 5,09 & 2,76 & 5,52 & 5,06 & 5,06 & 2,71 & 5,43 \\
\hline Levofloxacina & 3,66 & 14,65 & 3,27 & 13,07 & 3,25 & 12,98 & 3,09 & 12,34 & 3,05 & 12,19 & 3,31 & 13,23 \\
\hline \multicolumn{13}{|c|}{ REGNO UNITO } \\
\hline Ciprofloxacina & 4,94 & 4,94 & 2,22 & 4,44 & 4,51 & 4,51 & 3,62 & 7,25 & 5,41 & 5,41 & 3,81 & 7,63 \\
\hline Levofloxacina & 2,20 & 8,80 & 1,00 & 4,00 & 2,38 & 9,50 & 1,75 & 7,00 & 3,58 & 14,32 & ---- & ---- \\
\hline \multicolumn{13}{|l|}{ USA } \\
\hline Ciprofloxacina & 3,27 & 3,27 & 3,03 & 6,06 & 3,41 & 3,41 & 2,79 & 5,57 & 3,23 & 3,23 & 2,55 & 5,10 \\
\hline Levofloxacina & 1,69 & 6,77 & 2,11 & 8,44 & 1,69 & 6,77 & 2,01 & 8,04 & 1,66 & 6,63 & 1,87 & 7,46 \\
\hline
\end{tabular}

\section{Tabella 3}

Quantità di antibiotico mediamente consumata per ciclo di terapia antibiotica orale o parenterale con ciprofloxacina o levofloxacina in sei diverse nazioni negli anni 1999, $2000 e$ 2001. Le quantità sono espresse in unità ponderali (grammi) $e$ nell'equivalenti unità convenzionali del sistema DDD $(D D D$ ciprofloxacina orale $=$ 1000mg; DDD ciprofloxacina parenterale $=500 \mathrm{mg}$; DDD levofloxacina orale $=250 \mathrm{mg} ; D D D$ levofloxacina parenterale $=250 \mathrm{mg}$ ) 
ri a quelle dell'unità di misura DDD (1 grammo), mentre la forma parenterale viene usata mediamente a dosi medie giornaliere superiori alla relativa DDD $(0,5$ grammi). La levofloxacina in tutti i paesi considerati viene usata, sia per via orale sia per via parenterale, a dosi medie giornaliere nettamente superiori al valore della DDD $(0,25$ grammi sia per via orale che parenterale).

I dati riportati in tabella 3 evidenziano ancora una volta il significato e i limiti delle DDD come unità di misura e soprattutto come all'unità DDD non possa essere attribuito il significato di dose media prescritta o consumata.

In conclusione, avendo la possibilità di stimare indicatori basati sulle quantità realmente prescritte o consumate (numero di pazienti, numero di cicli terapeutici, durata di un ciclo, PDD e quantità di farmaco consumata per ciclo di terapia), gli indicatori basati sulla tecnica delle DDD risultano superflui perchè producono informazioni meno precise e non riferibili direttamente alla situazione clinica dei pazienti e ai relativi bisogni terapeutici.

\section{VALORIZZAZIONEMONETARIA DEI CONSUMI}

Il dati di consumo ottenuti mediante la tecnica delle DDD o la stima delle PDD vengono talvolta valorizzati in termini monetari per attuare analisi dei costi dell'assistenza farmaceutica e soprattutto dei trend della spesa farmaceutica in funzione dei pattern prescrittivi dei medici o più in generale delle modalità di consumo di singoli farmaci o di classi terapeutiche di farmaci. Nell' attuare questo tipo di valutazioni occorre avere ben presente il significato dei dati di consumo ottenuti con la tecnica delle DDD o con la stima delle PDD, al fine di evitare errori metodologici e interpretativi che potrebbero indurre i decisori a prendere scelte tecnicamente sbagliate (ad esempio nel decidere l'inclusione o l'esclusione di un prodotto in prontuari nazionali, regionali od ospedalieri).

La valorizzazione monetaria può sempre essere attuata, ma non sempre è utile o sensata e talvolta può essere addirittura fuorviante rispetto alle decisioni da prendere. Ad esempio, soprattutto nel caso di farmaci usati per brevi cicli, come gli antibiotici, non ha molto senso e crea inutili ulteriori confusioni stimare il costo del numero di DDD/1000 abitanti/anno o il costo del numero di DDD/1000 abitanti/die.

La valorizzazione monetaria di una DDD o di un grammo di antibiotico può essere fatta, ma deve servire esclusivamente come tappa intermedia per calcolare il costo di acquisto della quantità di farmaco consumata giornalmente o consumata in un intero ciclo di terapia. Sarebbe grave errore considerare il costo di una DDD o di un grammo in una valutazione farmacoeconomica comparativa finalizzata a determinare quale farmaco sia più conveniente tra le alternative considerate.

Quando la valutazione farmacoeconomica ha come obiettivo quello di determinare quale sia l'alternativa terapeutica più conveniente tra quelle disponibili nella prospettiva di un decisore, la semplice analisi dei costi, e soprattutto dei costi correlati a dati epidemiologici di utilizzo dei farmaci, non è adatta a risolvere il problema; per affrontare correttamente il problema della scelta dell'alternativa più conveniente e perseguire l'obiettivo di raggiungere la massima efficienza allocativa delle risorse, occorre utilizzare tecniche più complesse e sofisticate, come l'analisi costo/efficacia o l'analisi costo/utilità: per ogni alternativa considerata occorre valutare tutti i costi appropriati e le specifiche conseguenze sulla salute.

In questo tipo di valutazione i costi d'acquisto sono, nella maggior parte dei casi, solo una piccola quota dei costi totali da considerare. Inoltre, per poter attuare una valida analisi costo/efficacia, sia il consumo di risorse (costi) sia i benefici sulla salute vengono generalmente rilevati sul singolo paziente; quindi, mediante lo studio di un campione di pazienti, si ricavano le distribuzioni dei parametri di costo e dei parametri di efficacia con le loro tendenze centrali (media o mediana).

Nelle valutazioni farmaceoconomiche, in ogni caso, è indispensabile stimare le quantità di farmaco realmente prescritte dal medico o dispensate dal farmacista o consumate dal paziente, quindi valorizzare tali quantità in termini monetari.

Generalmente un farmaco è presente sul mercato in diverse formulazioni (per uso parenterale, orale, ecc.), livelli dose (es. 250mg, $500 \mathrm{mg}$ e $1000 \mathrm{mg}$ ) e confezioni (es 1 fiala, 10 compresse, 6 capsule). La stima delle quantità di farmaco consumate mediamente da un paziente nel corso di un ciclo di terapia o di malattia può essere attuata utilizzando l'unità di misura previsto dal sistema delle DDD o molto più semplicemente stimando la dose media giornaliera consumata (PDD) e la durata media del ciclo di terapia.

Ogni tipo di confezione ha un suo prezzo e non sempre il prezzo per unità di peso della sostanza è uniforme tra le varie confezioni. Inoltre, il costo d'acquisto del farmaco varia moltissimo in funzione di chi acquista e paga: il paziente, se acquista direttamente il farma- 
co, lo paga a prezzo pieno, il SSN rimborsa al farmacista territoriale il $97 \%$ del prezzo di listino per i farmaci inseriti in fascia A, l'ospedale acquista ad un prezzo non superiore al $50 \%$ del prezzo al pubblico.

La valorizzazione monetaria del numero di DDD o di PDD consumate in un ciclo terapeutico richiede una particolare attenzione a tutte queste variabili che regolano la formazione dei costi di acquisto dei farmaci ai vari livelli di organizzazione del sistema sanitario italiano.

La tabella 4 riporta alcuni valori monetari di unità fisiche o di indicatori di farmacoutilizzazione. I valori monetari sono espressi in euro e sono stati stimati applicando per semplicità il prezzo di vendita al pubblico, in vigore al $1^{\circ}$ ottobre 2002 , delle varie confezioni dei prodotti considerati.

Si deve notare, innanzitutto, che il costo/ DDD o il costo/grammo dipenda dal costo della confezione. Il costo/DDD o il costo/grammo riferito all' antibiotico risulta da una media ponderata che tiene conto delle differenze di prezzo per confezione e dalla percentuale di utilizzo delle singole confezioni.
Il volume di confezioni vendute in un anno sull'intero territorio nazionale ha un equivalente monetario che viene normalmente stimato ai fini di tenere sotto osservazione l'andamento della spesa farmaceutica. Questo parametro, tuttavia, non è particolarmente interessante ai fini farmacoeconomici, mentre è utilissimo per analizzare i trend del mercato farmaceutico.

Il costo/PDD è il parametro fondamentale dal punto di vista delle analisi farmacoeconomiche.

I dati riportati nella tabella 4 evidenziano come il valore monetario di una PDD sia esattamente identico al valore monetario del numero di DDD mediamente consumate in un giorno di terapia.

Confrontando il costo/PDD con il costo/ DDD di ciascun prodotto o di ciascun antibiotico emerge ancora una volta l'irrazionalità di utilizzare la DDD o il suo valore monetario come parametri di confronto in valutazioni farmacoeconomiche.

In particolare, emerge che i valori monetari delle PDD stimate per $\mathrm{i}$ prodotti a base di ciprofloxacina, di levofloxacina e di

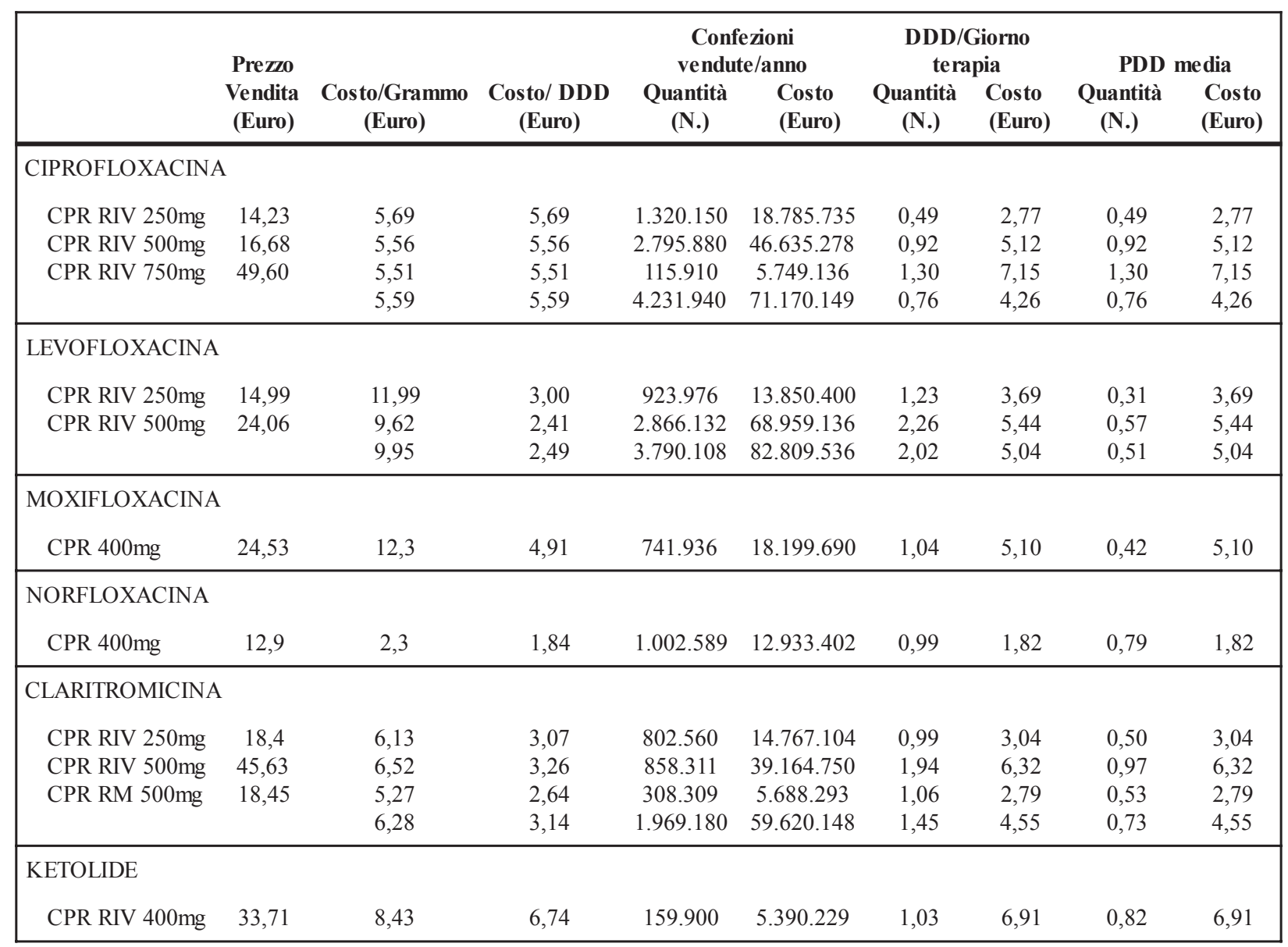

\section{Tabella 4}

Prezzo di vendita, costo/grammo, costo/DDD, costo totale annuale, costo/N. DDD/giorno terapia e costo/PDD di alcuni antibiotici in Italia in base ai prezzi di vendita in vigore al $1^{\circ}$ ottobre 2002. 
claritromicina variano nettamente rispetto ai corrispettivi valori monetari dell'unità di misura DDD.

Inoltre il valore monetario medio della PDD stimato per la ciprofloxacina è nettamente inferiore al valore medio monetario della relativa DDD. Al contrario, i valori monetari medi delle PDD stimati per la levofloxacina e la claritromicina sono nettamente superiori ai corrispettivi valori monetari medi delle relative DDD.

Si deve sottolineare come la PDD, analogamente al numero di DDD consumate, è una stima del consumo di un farmaco e non contiene in sè alcun riferimento ai risultati terapeutici ottenuti.

L'analisi dei costi attuata usando le PDD o il numero di DDD consumate deve essere interpretata in chiave descrittiva trasversale o longitudinale, ma non in chiave di analisi costi/efficacia. Solo nel caso si possa assumere con sufficiente robustezza che i consumi di due farmaci comportano risultati clinici equivalenti in termini quali-quantitativi, l'analisi dei costi può assumere la valenza di un' analisi "minimizzazione dei costi" (sottospecie dell'analisi costi/efficacia).

La valorizzazione monetaria dei consumi di farmaci è utile per valutare a livello di macroaree geografiche o assistenziali (nazione, regione, ASL, ospedale) l' andamento della spesa farmaceutica in relazione all' assistenza sanitaria erogata ai pazienti affetti da patologie (es. infettive) e per mettere a punto eventuali strategie di razionalizzazione della gestione dei pazienti. [23-25]

Si deve, tuttavia, sottolineare ancora una volta come questi dati non possano essere interpretati in termini di analisi costo/efficacia per due principali motivi: 1) non rappresentano il costo medio per paziente o per episodio di malattia; 2) non sono rapportati direttamente ai risultati clinici (successo, insuccesso) ma solo all'esposizione al farmaco.

\section{CONCLUSIONI}

La tipologia dei dati di consumo disponibili (fonte, metodo di rilevazione a campione e di estrapolazione statistica alla popolazione dei pazienti) condiziona la scelta del metodo per valutare e confrontare modalità di utilizzo dei farmaci e del loro impatto economico sul SSN e sulla società.

L'utilità e i limiti della tecnica delle DDD sono stati ripetutamente analizzati da vari autori negli ultimi anni e precise indicazioni sono reperibili anche sul sito WEB dell'OMS. [8,9] Ciò nonostante esiste ancora una diffusa in- certezza nell'utilizzo di tale tecnica e troppo spesso dobbiamo ancora registrare episodi di cattivo uso degli indicatori basati sulle DDD. In particolare, all'unità di misura DDD viene talvolta ancora attribuito il significato di dose media giornaliera prescritta/consumata o di dose raccomandata.

I parametri ricavabili con la tecnica delle DDD dai dati di vendita dei prodotti farmaceutici sono adatti per monitorare l'utilizzazione dei farmaci e l'evoluzione della spesa farmaceutica e per effettuare valutazioni farmacoeconomiche secondo l'approccio del così detto "paniere dei beni". [10]

Il metodo delle PDD è più diretto, indica le quantità medie giornaliere prescritte senza dover ricorrere a nuove unità di misura, differenti da farmaco a farmaco, consente di stimare senza complicazioni interpretative non solo la dose media giornaliera consumata, ma anche la dose media totale consumata per ciclo terapeutico ed eventuali altri indicatori di rilevanza clinica.

Tuttavia, per stimare le PDD e gli altri parametri derivati occorre rilevare una maggior quantità di informazioni rispetto ai semplici volumi di prescrizioni o di vendite.

Entrambi i metodi di stima consentono di evidenziare trend di utilizzo e di spesa e di confrontare intensità e modalità d'utilizzo in aree geografiche o in settori assistenziali differenti. In questo lavoro abbiamo presentato alcuni esempi adatti a illustrare l'utilizzo di queste tecniche per confrontare trend di utilizzo e di spesa di alcuni antibiotici e abbiamo sottolineato la preferenza a utilizzare le PDD, ogni qualvolta ciò sia possibile.

Tuttavia, gli indicatori ricavati con la tecnica delle DDD, ma anche le PDD e i parametri derivati, non sono utilizzabili direttamente in studi di farmacoeconomia orientati ad analizzare l'impatto di trattamenti farmacologici sull'efficienza allocativa delle risorse sanitarie. [13]

La massima efficienza allocativa dipende dalla scelta sistematica dell'alternativa maggiormente costo/efficace tra quelle disponibili. Per effettuare analisi costo/efficacia occorre stimare non solo i costi ma anche le conseguenze indotte dall'utilizzo di un farmaco. Inoltre occorre poter ricostruire, per ogni singolo paziente, l'intero percorso delle scelte e delle relative conseguenze per stabilire parametri affidabili di costo/efficacia.

Senza correlare il consumo dei farmaci e i relativi costi ai risultati clinici della terapia non è possibile stabilire quale sia il farmaco maggiormente costo/efficace e quindi decidere correttamente quale sia la scelta più conveniente e compatibile con le risorse sanitarie e sociali disponibili. 
L'unità di misura DDD viene usata per costruire diversi indicatori dell'intensità d'utilizzo dei farmaci. Possiamo stimare tassi d'utilizzo basati sul numero di DDD consumate per anno o per giorno e per residente o per paziente.

Il numeratore di questi tassi è la somma cumulativa di DDD consumate:

$$
\text { N. dosi unitarie dispensate }
$$

N. DDD consumate $=\frac{}{(\text { DDD/dose unitaria })}$

Dove:

- $\quad$ N. dosi unitarie dispensate $=\mathrm{N}$. confezioni $* \mathrm{~N}$. dosi unitarie per confezione

- DDD è il valore assegnato dall'OMS

- Dose unitaria è la quantità di farmaco presente in un'unità farmaceutica ed espressa nella stessa unità di misura della DDD.

Il rapporto DDD/dose unitaria indica quante unità farmaceutiche sono necessarie per realizzare una DDD del farmaco. Ad esempio, essendo la DDD della ciprofloxacina per via orale fissata a $1000 \mathrm{mg}$, per realizzare una DDD di questo antibiotico occorre consumare 4 compresse da $250 \mathrm{mg}$ oppure 2 compresse da $500 \mathrm{mg}$.

Si stima la somma delle DDD di un farmaco dispensate in un anno in un dato contesto d'utilizzo (ad esempio sull'intero territorio di una nazione). Il tasso di utilizzo per giorno si calcola dividendo il numero di DDD consumate in un anno per i 365 giorni dell'anno.

Quando sono disponibili i soli dati di vendita annuali, si possono stimare due diversi indicatori dell'intensità di utilizzo dei farmaci con significati epidemiologici differenti.

Un primo indicatore è il numero di DDD per 1000 residenti per anno. Questo tasso di utilizzo si ottiene dividendo il numero cumulativo di DDD consumate in un anno dalla popolazione considerata per il numero di abitanti della popolazione stessa, moltiplicato per 1000 .

$$
\text { N. DDD/1000 abitanti/anno }=\frac{\text { N. DDD cumulative/anno }}{\text { N. abitanti di una nazione }} * 1000
$$

Questo indicatore viene interpretato come giorni di trattamento (* 1000) che ogni residente potrebbe aver mediamente consumato in un anno, qualora tutti fossero stati trattati con una dose media giornaliera uguale alla DDD.

Ad esempio 5000 DDD/1000 abitanti/anno indica che il consumo è equivalente al trattamento di ogni abitante con un ciclo medio di terapia della durata di 5 giorni ogni anno. In realtà il valore numerico di questo indicatore non esprime il valore effettivo della durata media di trattamento, ma semplicemente un fattore parametrico di proporzionalità con tale grandezza: la durata media di trattamento dipende dal numero di pazienti trattati in una popolazione e dalla dose media giornaliera realmente consumata.

\section{RiquadroA}

Principali indicatori dell'intensità d'utilizzo dei farmaci derivati con la tecnica delle DDD.

Continua 
Un secondo indicatore è il numero di DDD consumate al giorno per 1000 residenti.

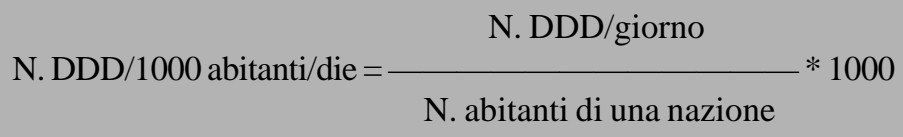

Questo indicatore stima in modo approssimativo la proporzione di soggetti di una popolazione trattati giornalmente con quel farmaco. Ad esempio $6 \mathrm{DDD} / 1000$ abitanti/die indica che mediamente lo $0,6 \%$ della popolazione è trattata giornalmente con quel farmaco.

Questa interpretazione, tuttavia, non è valida se la dose media consumata giornalmente si discosta significativamente dal valore teorico della DDD.

Negli studi di farmacoutilizzazione a livello ospedaliero basati sul volume delle prescrizioni si utilizza un indicatore che parametrizza il consumo dei farmaci ai giorni letto, ossia ai giorni di degenza in un dato periodo. L'indicatore generalmente usato è il numero di DDD/100 giorni letto. [18-22] La stima può essere fatta per ospedale o per reparto ed è utile per monitorare i comportamenti prescrittivi nei vari ambiti terapeutici dell'ospedale. [19, 21]

Quando i dati di consumo sono ricavati dal campionamento delle prescrizioni (es. ricette) è talvolta possibile stimare i giorni cumulativi di trattamento con un dato prodotto o farmaco in un anno. Conoscendo anche il numero di pazienti trattati si può ricavare una stima della durata media del trattamento

Sommatoria dei giorni di trattamento/anno

Durata media trattamento $($ giorni $)=\frac{\text { N. pazienti trattati/anno }}{\text { Som }}$

Dividendo il numero di DDD consumate per paziente in un anno per la durata media del trattamento si ottiene la stima del numero di DDD per giorno di trattamento, un indicatore di consumo che più facilmente può essere interpretato in funzione clinica

$$
\text { N. DDD/giorno di trattamento }=\frac{\text { N. DDD/paziente/anno }}{\text { durata media trattamento (giorni) }}
$$

Il suo valore dovrebbe essere uguale o vicino ad 1 se il farmaco fosse usato per la sua indicazione principale alla dose raccomandata: valori superiori o inferiori ad 1 possono indicare che il farmaco è invece usato principalmente per indicazioni diverse da quella principale e/o a dosi differenti, oppure che il farmaco è sottodosato o iperdosato quanto utilizzato nell'indicazione principale assunta come riferimento nell' unità di misura DDD.

Questo indicatore può essere aggiustato per età e sesso dei pazienti o per altre variabili cliniche che interessa tenere sotto controllo.

In caso ciò non sia richiesto la stima dell'indicatore si semplifica ed equivale al semplice rapporto tra numero di DDD anno e sommatoria dei giorni di terapia in un anno.

$$
\text { N. DDD/giorno di trattamento }=\frac{\text { N. DDD/anno }}{\text { Sommatoria giorni trattamento/anno }}
$$


La Prescribed Daily Dose (PDD) è un valore medio della quantità di farmaco (in unità ponderali) prescritta (quindi presuntivamente consumata) giornalmente in un dato ambito territoriale o assistenziale.

Il valore della PDD si ottiene dal rapporto di due tassi:

$$
\mathrm{PDD}=\frac{\text { Quantità di farmaco (in peso) prescritto in un anno/N. pazienti trattati }}{\text { Sommatoria di giorni di trattamento in un anno/N. pazienti trattati }}
$$

Questi tassi possono essere stimati per fasce d'età e per sesso o per altri parametri che interessa tenere sotto controllo.

Qualora non siano necessarie analisi per stratificazione di popolazione il valore della PDD si ottiene più semplicemente dividendo la quantità di farmaco prescritta in un anno per la sommatoria dei giorni di trattamento nell'anno.

$$
\mathrm{PDD}=\frac{\text { Quantità di farmaco (in peso) prescritto in un anno }}{\text { Sommatoria di giorni di trattamento in un anno }}
$$

La PDD è un valore statistico (media) della dose di farmaco realmente prescritta in una popolazione di pazienti. Pertanto, è un indicatore preferibile quando si intenda confrontare il consumo ed eventualmente i costi di farmaci alternativi. [12]

\section{Riquadro B}

Definizione e calcolo delle PDD.

\section{BIBLIOGRAFIA}

1. Engel A, Siderius P. The consumption of drugs. Report on a study 1966-1967. World Health Organisation, Regional Office for europe, Copenaghen (EURO 3101), 1968.

2. Bergman U, Elmes P, Halse M, et al. The measurement of drug consumption. Drugs for diabetes in Northern Ireland, Norway and Sweden, Eur j Clin Pharmacol 8 : 83-89, 1975.

3. Lunde PKM, Baksaas I, Halvorsen T, et al. The methodology of drug utilization studies. In : Bergman U et al eds. «Studies in drug utilisation ». WHO Regional Publications. Europe Series, Copenaghen 8 : 17-28, 1979.

4. Agenas I, Andrew M. Sales statistics. In : « Nordic Statistics on Medicines 1981-1983, 1: NLN Publication No 13, Nordic Council on Medicines, Uppsala, 1983.

5. Serradell J, Bjornson DC, Hartzema AG. Drug utilization study methodologies: national and international perspectives. DICP 21: 994-1001, 1987.

6. Wessling A, Boethius G, Measurement of drug use in a defined population. Evaluation of the Defined daily Dose (DDD) Methodology. Eur J Clin Pharmacol 39. 207-210, 1990.

7. Dukes G (Ed). Drug utilization studies. Methods and uses. WHO Regional Pubblications. European series No 45. WHO 1993, ISBN 9289013087.

8. WHO. Guidelines for ATC classification and DDD assignment. WHO Collaborating Centre for Drug Statistics Methodology. Oslo, Norway 2001. ISBN 8290312326 (www.whocc.no/atcddd/)

9. WHO. Anatomical Therapeutic Chemical (ATC) Classification Index with Defined Daily Doses (DDDs). WHO Collaborating Centre for Drug Statistics Methodology. Oslo, Norway January 2002. (www.whocc.no/atcddd/)

10. Brenner G. Methods to assess health economics aspects of drug utilisation. Acta Med Scand 721: (Suppl): 31-35, 1987. 
11. Cooke J. Drug utilisation research. Int J Pharm Pract 1: 5-9, 1991.

12. Merlo J, Wessling A, Melander A. Comparison of dose standard units for drug utilsation studies. Eur J Clin Pharmacol 50: 27-30, 1996.

13. Clarke KW, Gray D. The Defined Daily Dose as a tool in pharmacoeconomics. Advantages and limitations. Pharmacoeconomics 7: 280-283, 1995.

14. Bergman U, Christenson I, Jansson B et al. Auditing hospital drug utilisation by means of Defined Daily Doses per bed-day. A methodological study. Eur J Clin Pharmacol 17: 183-187, 1980.

15. Ronning M, Blix HS, Harbo BT, et al. Different versions of the anatomical therapeutic chemical classification system and the defined daily dose - are drug utilisation data comparable? Eur J Clin Pharmacol 56: 723-727, 2000.

16. Cosentino M, Leoni O, Banfi F, et al. An approach for the estimation of drug prescribing using the defined daily dose methodology and drug dispensation data. Theoretical considerations and practical applications. Eur $\mathrm{J}$ Clin Pharmacol 56: 513- 517, 2000.

17. Resi D, Castelvetri A, Vaccheri A, Montanaro N. The therapeutic course as a measure complementary to Defined Daily Doses when studying exposure to antibacterial agents. Eur J clin Pharmacol 57: 177-180, 2001.

18. Hekster YA, Vree TB, Goris RJA, et al. The defined daily dose per 100 bed-days as a unit of comparison and a parameter for studying antimicrobial drug use in a university hospital. A retrospective study of the effects of guidelines and audit on antimicrobial drug use. J Clin Hosp Pharm 7: 251-260, 1982.

19. Scroccaro G, Marini P, Pedrini A, et al. Studio epidemiologico sul consumo in DDD degli antibiotici in undici ospedali italiani. Giorn It Farmacia Clin 3: 65-75, 1989.

20. Fletcher CV, Metzler D, Borchardt-Phelps P, et al. Patterns of antibiotic use and expenditures during 7 years at a university hospital. Pharmacotherapy 10: 199-204, 1990.

21. Messori A. Analisi dei consumi ospedalieri. Giorn It Farmacia Clin 7: 123-133, 1993.

22. Kiivet RA, Dahl ML, Llerena A, et al. Antibiotic use in 3 european university hospitals. Scand J Infect Dis 30: 277280, 1998.

23. Maxwell M, Heaney D, Howie JGR, et al. General practice fundholding: observations on prescribing patterns and costs using the defined daily dose method. BMJ 307: 1190-1194, 1993.

24. Maxwell M, Howie JGR, Pryde CJ. A comparison of three methods of setting prescribing budgets, using data derived from defined daily dose analyses of historic patterns of use. Br J Gen Pract 48: 1467-1472, 1998.

25. Lucioni C. Farmacoeconomia: strumento di razionalizzazione delle scelte pubbliche. Giorn It Farmacia Clin 7 : 119-122, 1993. 